\title{
Morphological and mucus secretion criteria for differential diagnosis of solitary ulcer syndrome and non-specific proctitis
}

\author{
M EHSANULLAH, MI FILIPE, B GAZZARD \\ From the Departments of Histopathology and Gastroenterology, Westminster Hospital, \\ London SWIP 2PP
}

SUMMARY Over a four-year period 21 cases of solitary ulcer syndrome (SUS) were studied for their $\tilde{N}_{\mathscr{\Omega}}$ clinical, histological, and mucus secretion patterns and compared with histological and mucus 은 secretion patterns of 78 cases of non-specific proctitis collected over the same period. Normal mucus composition was found in non-specific proctitis while abnormalities of mucins with predominance of sialomucins were associated with SUS. Although histology remains the most important investigation in the diagnosis of SUS, mucin changes provide valuable additional evidence.

Solitary ulcer syndrome (SUS) and non-specific proctitis both present with symptoms which are common to most anorectal diseases. ${ }^{1-3}$ The sigmoidoscopic appearance and particularly the histological features of the rectal mucosa are used to differentiate these two conditions. We investigated the mucin-staining patterns in the rectal biopsies of patients with these diseases to see if this provided useful additional information.

\section{Patients and methods}

The clinical features were studied closely and the histology of the rectal mucosa and the mucin secretion patterns were compared, in 78 patients with non-specific proctitis and 21 cases of SUS, seen over a four-year period (1975-9). Patients had nonspecific proctitis if on sigmoidoscopy inflammation was limited to the rectum with normal mucosa above this and a normal barium enema. All patients had a rectal biopsy which showed a variable inflammatory infiltrate in the lamina propria by mononuclear or mixed cells with occasional crypt abscesses. Distorted crypts and hyperplasia were also observed. There was no evidence of ulcerative colitis, Crohn's disease or an infective type of proctitis. A clinical diagnosis of SUS was made in patients who had a normal barium enema but in whom rectal examination revealed induration and sigmoidoscopy showed

Accepted for publication 22 May 1981 either an ulcer without other evidence of inflammation or a localised area of hyperaemia with abnormal perineal descent or prolapse of the rectal mucosa.

All rectal biopsies were routinely fixed in $10 \% \stackrel{2}{\approx}$ formol saline and paraffin sections were stained with $\stackrel{\mathbb{Q}}{\varrho}$ haematoxylin and eosin and van Gieson's stain. To $\overrightarrow{\vec{O}}$ assess the amount and types of mucins the following $\frac{3}{3}$ techniques were used: periodic acid-Schiff (PAS) ${ }^{4}$ and high iron-diamine-alcian blue (HID-AB) ${ }^{5}$ which distinguish between neutral, sulpho- and sialomucins. The histologica! criteria for SUS ${ }^{1}$ were based on the presence of mucosal ulceration, increased fibroblasts in the lamina propria with minimal inflammation and thickened muscularis mucosae with tendency of individual fibres to point toward the lumen. Distorted crypts, hyperplasia and mucus depletion were seen in some cases.

The histology of 21 patients with SUS was reviewed without the knowledge of previous results $N$ and other clinical details. All cases were then classified into three groups:

A: Patients with definite clinical diagnosis of SUS supported by accepted histological criteria.

B: Patients who were suspected to have SUS on clinical grounds but the histological features were equivocal.

C: Patients with clinical appearances suggestive of SUS, in whom the histology showed non-specific changes.

In the normal rectal mucosa, mucus secretion contains predominantly sulphomucins (normal mucin 
Table 1 Histological features and mucin patterns in 21 cases of solitary ulcer syndrome (SUS)

\begin{tabular}{|c|c|c|c|c|c|c|c|c|c|c|c|c|c|c|c|c|c|c|c|c|c|}
\hline \multirow[t]{2}{*}{${ }^{*}$ Histology } & \multicolumn{21}{|c|}{ Cases } \\
\hline & 1 & 2 & 3 & 4 & 5 & 6 & 7 & 8 & 9 & 10 & 11 & 12 & 13 & 14 & 15 & 16 & 17 & 18 & 19 & 20 & 21 \\
\hline \multirow{9}{*}{$\begin{array}{l}\text { Lamina propria } \\
\text { Fibroblasts } \\
\text { Smooth muscle } \\
\text { Inflammation } \\
\text { Mucosal erosion } \\
\text { Crypt distortion } \\
\text { Epithelial hyperplasia } \\
\text { Thickening of } \\
\text { muscularis mucosae } \\
\text { Sialomucins } \\
\text { Group A, B, C } \\
\text { (see text) }\end{array}$} & & & & & & & & & & & & & & & & & & & & & \\
\hline & + & + & & + & + & + & & & + & + & $\div$ & + & + & + & + & + & + & + & + & + & + \\
\hline & 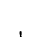 & + & & + & $+?$ & $+?$ & & & & + & & $+?$ & + & & + & + & + & 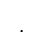 & + & & \\
\hline & + & & + & + & & $+?$ & + & + & + & + & & & + & + & $\begin{array}{l}+ \\
+\end{array}$ & & + & $\begin{array}{l}+ \\
+\end{array}$ & & + & \\
\hline & & + & & + & + & & + & & & + & + & & + & + & + & + & + & + & & & \\
\hline & & + & & + & & & + & & & & & & + & & & & + & & + & & + \\
\hline & + & & & & & & & & & & & + & & & & & & & + & & \\
\hline & & + & & + & & + & + & + & & + & & + & + & + & + & + & + & + & + & & \\
\hline & C & B & C & $\mathbf{A}$ & B & B & C & C & C & $\mathbf{A}$ & C & B & A & B & $\mathbf{A}$ & B & $\mathbf{A}$ & B & $\mathbf{A}$ & C & C \\
\hline
\end{tabular}

$+=$ present.

$?=$ doubtful.

* = Rutter and Riddell (1975). ${ }^{1}$

pattern) or both sulpho- and sialomucins with the predominance of sulphomucins (mixed pattern). The pattern was considered abnormal if an excess of sialomucins was seen.

\section{Results}

Among the patients who suffered from SUS common symptoms were: rectal and lower abdominal pain; altered bowel habit; increased frequency being commoner than constipation; and passage of mucus rectally which sometimes took the form of rectal discharge. Rectal bleeding was found in 12 cases, single ulcer was seen in nine cases and four patients had a history of attending a psychiatric clinic with depression or anxiety. History of coexisting anorectal disease such as fissure in ano, fistulae, piles, or rectal prolapse was found in 10 patients. External prolapse of the rectal mucosa was seen in six cases. Patients with non-specific proctitis had a similar clinical picture except a history of rectal bleeding was invariable. Of 78 patients with non-specific proctitis, 12 had a history of attending a psychiatric clinic and none of them had an associated anorectal disease.

The histological features for each of the SUS patients are shown in Table 1 . In the 78 cases of non-specific proctitis the morphological changes consisted in hyperaemia, mild to severe inflammatory infiltrate in the lamina propria including neutrophils and formation of crypt abscesses in a few cases, associated or not with decreased mucus secretion, and crypt distortion. In none of the specimens was there erosion, significant increase in fibroblasts or the presence of smooth muscle fibres in the lamina propria. Epithelial hyperplasia was not a feature. No specific causes for the proctitis could be found and both ulcerative colitis and Crohn's
Table 2 Distribution of mucins in solitary ulcer syndrome (SUS) and in non-specific proctitis

\begin{tabular}{ccl}
\hline Solitary ulcer syndrome & Normal mucin pattern & Sialomucins \\
\hline Group A & 0 & 6 \\
B & 1 & 6 \\
C & 6 & 2 \\
Non-specific proctitis & 76 & 2 \\
\hline
\end{tabular}

For A, B, and C see text.

disease were excluded.

When the mucus secretion pattern of SUS and non-specific proctitis was compared, marked differences in mucin composition appeared (Table 2).

Mucin secretion in rectal biopsies from patients with SUS was frequently abnormal (Tables 1 and 2). Sialomucins were predominant in all cases in group A (Fig. 1) and in six out of seven cases in group B (the latter patients had suggestive clinical features of SUS but equivocal histology). In contrast, patients in group $\mathrm{C}$ showed a normal mucin pattern in six out of eight cases. It is interesting to note that three patients in group A and B who had e:icess sialomucins in the initial biopsies showed a 1lormal histology and normal mucins, on subsequent rectal biopsies when they were in remission.

In non-specific proctitis, the pattern of mucin secretion was normal in all but two cases (Figs. 2 and 3 ; Table 2). On careful review, one of these two cases had a single ulcer seen on sigmoidoscopy without surrounding inflammation and almost certainly had SUS while the second patient showed gross perineal descent.

Mucin secretion was reduced (visual assessment) in a small number of patients with both non-specific proctitis (10 in 72) and SUS ( 2 in 21$).$ 

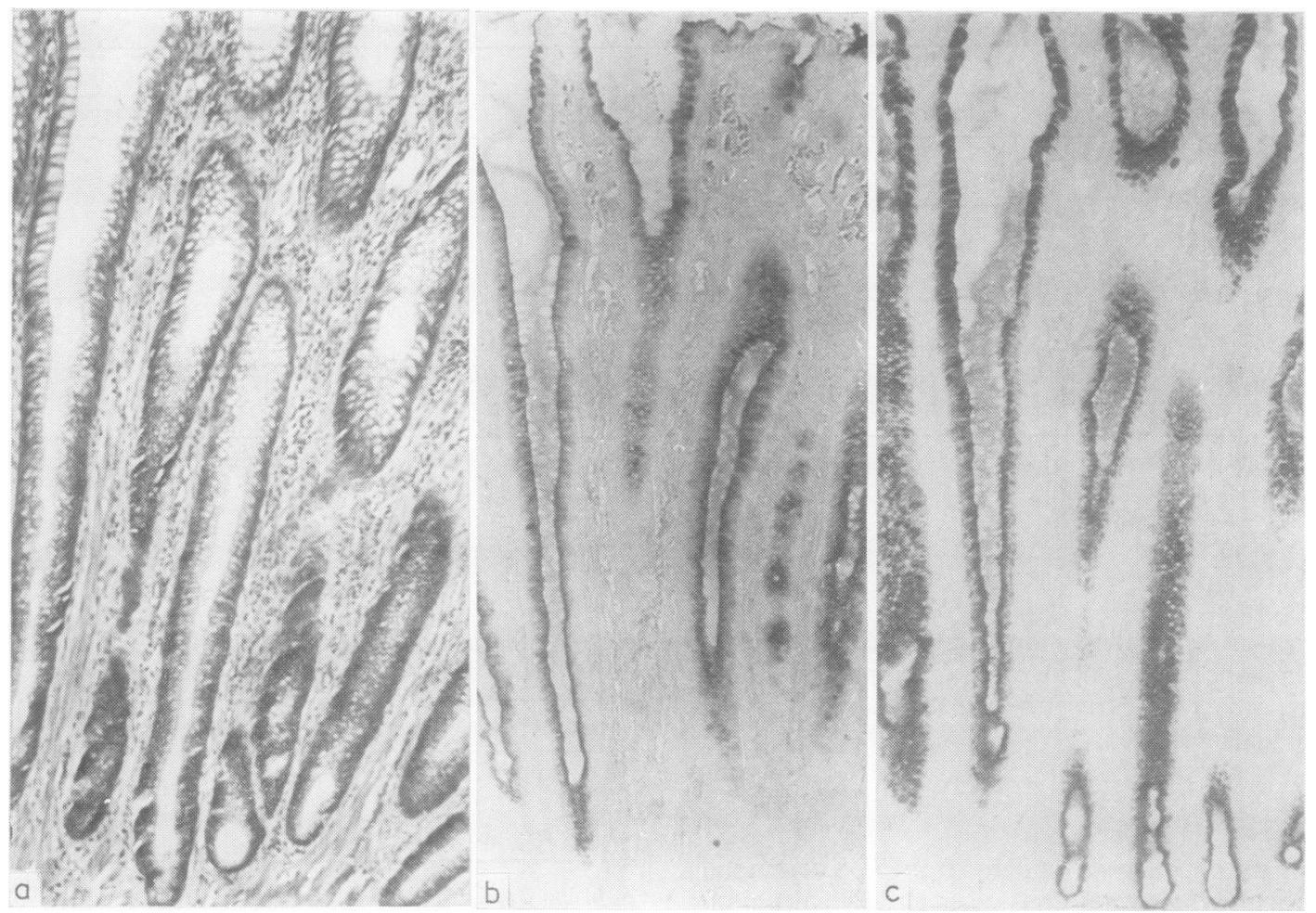

Fig. 1 Rectal mucosa in solitary ulcer syndrome showing (a) crypt hyperplasia, moderate mucin depletion, increased fibroblasts and muscle fibres pointing toward the lumen (haematoxylin and eosin), (b) mucin contains small anounts of sulphated material revealed by weak high iron-diamine staining and $(c)$ a higher proportion of sialomucins shown in a parallel section stained by the high iron-diamine-alcian blue method. Compare intensity of staining in $b$ with Figs. $2 b$ and $3 b \times 80$.

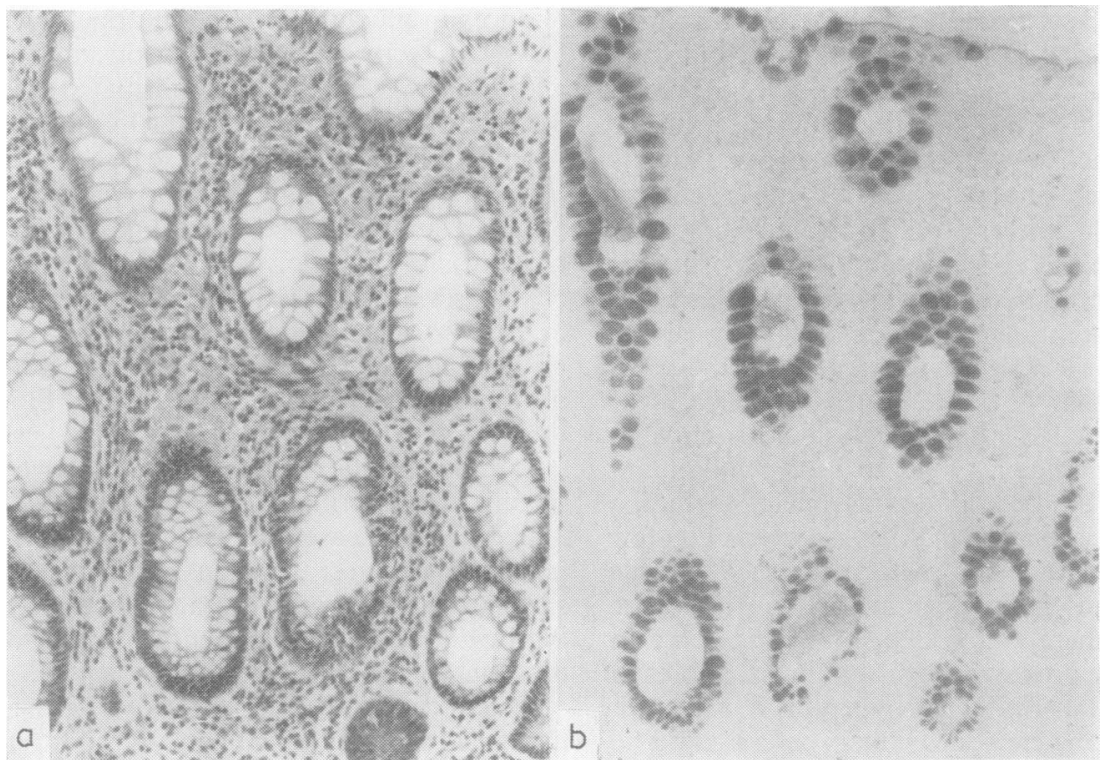

Fig. 2 Rectal mucosa in active non-specific proctitis showing (a) inflammation in the lamina propria with formation of a small crypt abscess (haematoxylin and eosin), and (b) preservation of mucin secretion which consists mainly of sulphomucins stained strongly with high iron-diamine $\times 130$. 


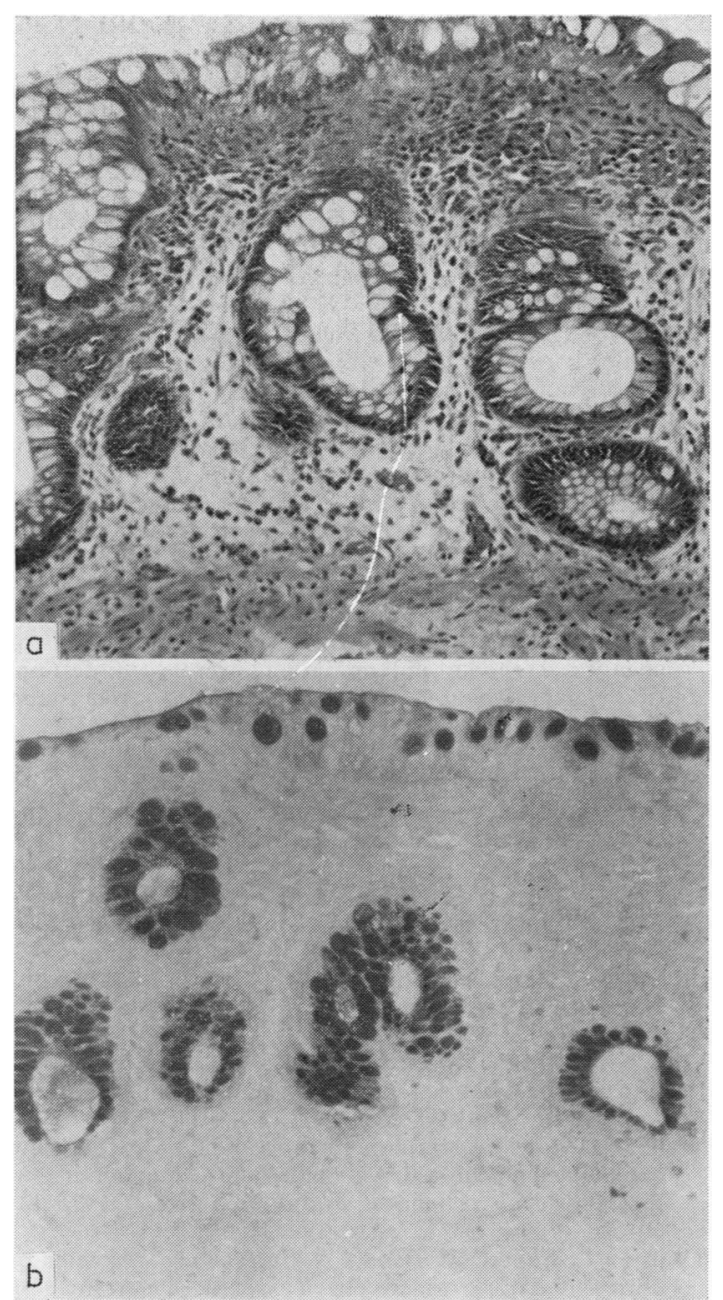

Fig. 3 Rectal mucosa in quiescent non-specific proctitis showing (a) distorted crypts in a background of little inflammation (haematoxylin and eosin), (b) mucins consist predominantly of sulphomucins stained strongly with high iron-diamine $\times 130$.

\section{Discussion}

From our observations it is clear that SUS and non-specific proctitis present with very similar symptoms, although there was an increased incidence of psychiatric illness and anorectal diseases such as piles, prolapsed rectum, fissure and fistulae in patients with solitary ulcer. The latter fact was also commented upon by Rutter and Riddell ${ }^{1}$ and Rutter. ${ }^{2}$ The value of sigmoidoscopy and rectal biopsy in the diagnosis of SUS is well established ${ }^{1-3}$ but there remain a number of cases in which histological appearances are not conclusive. The presence of sialomucins further supports the diagnosis of SUS in the presence of suggestive clinical features and equivocal histology as in patients in group B. The differences in mucin patterns with predominance of sialomucins in SUS in contrast with a normal mucus secretion in non-specific proctitis, helps in the differential diagnosis of these two conditions.

At present, the interpretation of the mechanism(s) involved in the mucin changes observed in SUS can only be speculative, as the cause of this entity is as yet unknown.

Some of the histological features, such as tall crypts, high mitotic activity with elongation of the proliferative zone, and decreased mucus secretion but no atypia, suggest a reactive hyperplastic process to mucosal damage. Ischaemia, trauma and alterations in autonomic nerve control have been implicated as possible factors. ${ }^{12}$ These factors may alter the composition of colonic mucus either by a direct effect on glycoprotein synthesis ${ }^{6} 7$ and/or indirectly through increased proliferative cell capacity. ${ }^{8-10}$ It is known that changes in glycoprotein synthesis take place along the crypt in parallel with cell differentiation;1112 neutral mucins or incomplete glycoproteins being synthesised by immature cells which later mature with the addition of acid radicals to the carbohydrate chain.

In SUS the higher proportion of immature cells within the enlarged proliferative crypt zone, possibly associated with decreased or selective uptake of glycoprotein precursors due to ischaemia, could explain the variations in the mucin composition described. Although the role of injury in the quantitative and qualitative changes in mucus production seems valid, ${ }^{1314}$ it fails to explain normal patterns of mucus secretion in other conditions causing reactive hyperplasia-for example, (a) in idiopathic proctitis even when inflammation is severe; (b) in reactive as opposed to dysplastic epithelia in ulcerative colitis $; 15$ (c) in reactive hyperplasia at the anastomotic site in rats which had undergone colonic surgery. ${ }^{16}$ Ischaemia may play a role in the particular mucin changes found in SUS. Mucin studies in ischaemic lesions may help to clarify this point.

Although changes of mucin pattern do not occur in most cases of reactive hyperplasia they have been reported in colonic mucosa in association with malignancy. ${ }^{15}$ 17-21 Increased sialomucins were observed in the "transitional" mucosa adjacent to carcinoma and in patches of uninvolved mucosa distant from it;151722 in familial polyposis coli, ${ }^{23}$ and in rats with dimethylhydrazine-induced colorectal carcinoma. ${ }^{1624}$ These findings strongly suggest an association between abnormal glycoproteins and carcinogenesis, and is supported by biochemical 
data. ${ }^{25-29}$

Solitary ulcer syndrome is the first condition, not associated with malignancy, which shows predominance of sialomucins. This may raise doubts about the value of excess sialomucins as a marker of colonic malignancy. We believe that this is not the case.

The colonic epithelial cells produce a sulphated glycoprotein through a complex process of synthesis involving many sugar precursors and specific enzymes. ${ }^{30}$ Many factors can alter this process to produce incomplete or abnormal glycoproteins. At present histochemical methods used to study these changes are of low specificity and only distinguish between neutral, sulphated and acid non-sulphated sialomucins but give no information on the other components of the carbohydrate molecule. Further investigation is needed to find a profile of the changes which may occur in the various sugars and sialic acid derivatives in different conditions, before the value of mucin patterns to differentiate a reactive from a neoplastic process can be firmly established.

As a conclusion, variations in mucin composition seem to be a useful parameter to differentiate SUS from non-specific proctitis, and may also help in the diagnosis of SUS when histology is equivocal.

We are thankful to Cancer Research Campaign and Westminster Research Trust for financial support and Mrs $\mathrm{K}$ Matharu for secretarial assistance and help. We are grateful to our colleague $\mathrm{Dr}$ AC Branfoot for his comments.

\section{References}

1 Rutter KRP, Riddell RH. The solitary ulcer syndrome of rectum. Clin Gastroenterol 1975;4:505-30.

${ }^{2}$ Rutter KRP. Solitary rectal ulcer syndrome. Proc $R$ Soc Med 1975;68:22-7.

${ }^{3}$ Madigan MR, Morson BC. Solitary ulcer of the rectum. Gut 1969;10:871-81.

4 Pearse AGE. Histochemistry-theoretical and applied. Vol 1, 3rd ed. London: Churchill-Livingstone, 1968.

${ }^{5}$ Spicer SS. Diamine methods for differentiating mucosubstances histochemically. J Histochem Cytochem 1965; 13:211-34.

6 Florey HW. The secretion of mucins and inflammation of mucous membranes. In: Lord Florey, ed. General pathology. 4th ed. London: Lloyd Luke (Medical Books), 1970:195.

' Smith B, Butler M. The autonomic control of colonic mucin secretion in the mouse. Br J Exp Pathol 1974;55: 615-21.

${ }^{8}$ Sprinz H. Factors influencing intestinal cell renewal. Cancer 1971;28:71-4.

${ }^{9}$ Brackett KA, Townsend SF. Regeneration of colonic mucosa in the rat. $J$ Morphol 1980;163:203-15.

10 Tutton PJM, Barkla DH. Neural control of colonic cell proliferation. Cancer 1980;45:1172-7.

$"$ Neutra MR, Richard JG, Trier JS. Glycoprotein synthesis, transport and secretion by epithelial cells of human rectal mucosa. Normal and cystic fibrosis. Lab Invest 1977;36: 535-46.

${ }^{12}$ Lamont JT, Ventola A. Galactosyltransferase in fetal, neonatal and adult colon: relationship to differentiation. Am J Physiol 1978;235:E213-17.

${ }^{13}$ Isaacson P, Attwood PRS. Failure to demonstrate specificity of the morphological and histochemical changes in mucosa adjacent to colonic carcinoma (transitional mucosa). J Clin Pathol 1979;32:214-8.

${ }^{14}$ Rhatigan RM, Sappos RO. Mucosal hyperplasia in colonic diverticula. Histopathology 1979;3:153-60.

${ }^{15}$ Filipe MI, Branfoot AC. Mucin histochemistry of the colon. In: Morson BC, ed. Current topics in pathology. Heidelberg: Springer-Verlag, 1976:143-78.

${ }^{16}$ Filipe MI, Scurr JH, Ellis H. Effects of faecal stream on experimental carcinogenesis. Morphological and histochemical changes. Cancer (in press).

17 Filipe MI, Branfoot AC. Abnormal patterns of mucus secretion in apparently normal mucosa of large intestine with carcinoma. Cancer 1974;34:282-90.

${ }^{18}$ Filipe MI. Mucins in the human gastrointestinal epithelium: a review. Invest Cell Pathol 1979;2:195-216.

19 Culling CFA, Reid PE, Burton JD, Dunn WL. A histochemical method cf differentiating lower gastrointestinal tract mucins from other mucins in primary or metastatic tumours. J Clin Pathol 1975;28:656-8.

${ }^{20}$ Culling CFA, Reid PE, Worth AJ, Dunn WL. A new histochemical technique of use in the interpretation and diagnosis of adenocarcinoma and villous lesions in the large intestine. J Clin Pathol 1977;30:1056-62.

${ }^{21}$ Dawson PA, Patel J, Filipe MI. Variations in sialomucins in the mucosa of large intestine in malignancy: a quantimet and statistical analysis. Histochem $J$ 1978;10:55972.

${ }^{22}$ Greaves P, Filipe MI, Branfoot AC. Transitional mucosa and survival in human colorectal cancer. Cancer 1980; 46:764-70.

${ }^{23}$ Filipe MI, Mughal S, Bussey HJ. Patterns of mucus secretion in the colonic epithelium in familial polyposis. Invest Cell Pathol 1980;3:329-43.

${ }^{24}$ Filipe MI. Mucous secretion in rat colonic mucosa during carcinogenesis induced by dimethylhydrazine: a morphological and histochemical study. Br J Cancer 1975;32: 60-77.

${ }^{25}$ Warren L, Fuhrer JP, Buck CA. Surface glycoproteins of normal and transformed cells: a difference determined by sialic acid and a growth-dependent sialytransferase. Proc Natl Acad Sci 1972;69:1838-42.

${ }^{26} \mathrm{Kim}$ YS, Isaacs R, Perdomo JM. Alterations of membrane glycopeptides in human colonic adenocarcinoma. Proc Natl Acad Sci 1974;71:4869-73.

27 Lamont JT, Isselbacher KJ. Alterations in glycosyltransferase activity in human colonic cancer. $J$ Natl Cancer Inst $1975 ; 43: 53-6$.

${ }^{28}$ Gorman TA, Lamont JT. Glycoprotein synthesis and secretion in human colon cancers and normal colonic mucosa. Cancer Res 1978;38:2784-9.

29 Rogers CM, Cooke KB, Filipe MI. The sialic acids of human large bowel mucosa: $\mathrm{O}$-acylated variants in normal and malignant states. Gut 1978;19:587-92.

${ }^{30}$ Gottschalk A. Definition of glycoproteins and their delimitation from other carbohydrate-protein complexes. In: Glycoproteins: their composition, structure and function. London: Elsevier, 1966.

Requests for reprints to: Dr MI Filipe, Department of Histopathology, Westminster Medical School, Udall Street Laboratories, Udall Street, London SW1P 2PP, England. 